\title{
Anti-enteric Neuronal Antibodies and the Irritable Bowel Syndrome: Are They Really the Accused?
}

TO THE EDITOR: I read with immense interest the fascinating article entitled "Anti-enteric neuronal antibodies and the irritable bowel syndrome" by Wood et al, ${ }^{1}$ which was published in January 2012 issue of Journal of Neurogastroenterology and Motility. The authors have demonstrated that almost $90 \%$ of IBS patients exhibited positive anti-enteric neuronal antibodies which stained the nucleus and cytoplasm of neurons in the enteric nervous system (ENS). Furthermore they also performed protein microarray analysis detecting anti-enteric neuronal antibodies reactivity for autoantigens in the serum of IBS patients. The antigens recognized by these antibodies included a nondescript ribonucleoprotein (RNP-complex), small nuclear ribonuclear polypeptide A and Ro-5,200 kDa. The authors concluded that IBS was probably an autoimmune disease and symptoms in some IBS patients might be the detrimental effect of the anti-enteric neuronal antibodies to the enteric neurons.

The pathophysiology of IBS is complex and multifactorial, including abnormal motility, visceral hypersensitivity, abnormal gas production and expulsion, food intolerance or allergy, inflammation, intestinal dysbiosis, and stress. ${ }^{2,3}$ Immune interactions of the ENS are currently receiving substantial attention in gastrointestinal motility disorders including IBS. Inflammatory neuropathy of the ENS (enteric ganglionitis) brings about neuronal dysfunction, degeneration, and even results in a complete loss of enteric neurons. ${ }^{4}$ Enteric ganglionitis can be either primary or secondary to a wide range of diseases, such as paraneoplastic, infectious, and neurological disorders.

There is a limited number of studies regarding the role of antibody against the enteric neurons on gastrointestinal motility disorders. Törnblom et $\mathrm{al}^{5}$ demonstrated only a small number (2 out of 12) IBS patients with inflammatory enteric neuropathy had antibodies directed towards neuronal ion channels (anti-voltage-gated potassium channels antibodies and anti- $\alpha 3$-acetylcho- line receptor antibodies). Based on the high seropositive rate of anti-enteric neuronal antibodies in their IBS patients, Wood et $\mathrm{al}^{1}$ concluded that an autoimmune degenerative neuropathy and loss of neurons in the ENS might play a role in a subset of IBS patients. There are several shortcomings of this study. First, there is no histopathologic evidence of enteric neuropathy to correlate with the serology. Second, there is no available data of protein microarray analysis in control sera. Third, the presence of anti-enteric neuronal antibodies might not be attributable to an autoimmune process. It has been demonstrated in rats that herpes simplex viruses are stored in myenteric ganglia of the gastrointestinal tract. ${ }^{6}$ Furthermore, a high percentage of control sera (almost 60\%) in Wood's study contained anti-enteric neuronal antibodies. Therefore the presence of those antibodies might have been caused by residues after previous viral infection.

In conclusion, whether presence of the anti-enteric neuronal antibodies is directly linked to gastrointestinal symptoms in patients with IBS or it is just an epiphenomenon, warrants further research.

\section{Voranush Chongsrisawat \\ Department of Pediatrics, Faculty of Medicine Chulalongkorn University, Bangkok, Thailand}

1. Wood JD, Liu S, Drossman DA, Ringel Y, Whitehead WE. Anti-enteric neuronal antibodies and the irritable bowel syndrome. J Neurogastroenterol Motil 2012;18:78-85.

2. Spiller R, Garsed K. Postinfectious irritable bowel syndrome. Gastroenterology 2009;136:1979-1988.

3. Alonso C, Guilarte M, Vicario M, et al. Maladaptive intestinal epithelial responses to life stress may predispose healthy women to gut mucosal inflammation. Gastroenterology 2008;135:163-172.

4. De Giorgio R, Guerrini S, Barbara G, et al. Inflammatory neuropathies of the enteric nervous system. Gastroenterology 2004;126: 1872-1883.

5. Törnblom H, Lang B, Clover L, Knowles CH, Vincent A, Lindberg 
G. Autoantibodies in patients with gut motility disorders and enteric neuropathy. Scand J Gastroenterol 2007;42:1289-1293.

6. Takase H, Yamamura E, Murakami Y, Ikeuchi T, Osada Y. Gastrointestinal invasion by herpes simplex virus type 1 inoculated cutaneously into the immunosuppressed mice. Arch Virol 1994;134:97-107.

\section{Conflicts of interest: None.}

\section{Corrigendum}

Physicians and Patients Measure Different Dimension on Assessment for Gatroesophageal Reflux Diseaserelated Symptoms

Juan Carlos Lopez-Alvarenga, Sergio Sobrino-Cossio, Ronnie Fass and Jose A Vargas-Romero

(J Neurogastroenterol Motil 2011;17:381-386; http://dx.doi.org/10.5056/jnm.2011.17.4.381)

Title of the paper was mis-typed. The correct title is "Physicians and Patients Measure Different Dimension on Assessment for Gastroesophageal Reflux Disease-related Symptoms." And title of the figure was incorrectly written. The correct title of the figure is "Symptoms improvement after 4 weeks of treatment with pantoprazole magnesium. (A) It shows patient evaluation with ReQuest $^{\mathrm{TM}}$. (B) It shows physician evaluation with a structured interview, despite the lack of correlation both measurements improved significantly with treatment. Both assessments showed significant amelioration of symptoms $(P<0.05$ for all variables)." 